Pacific

Journal of

Mathematics

\title{
VANISHING VISCOSITY IN THE PLANE \\ FOR NONDECAYING VELOCITY AND VORTICITY, II
}

Elaine CozZI 


\title{
VANISHING VISCOSITY IN THE PLANE FOR NONDECAYING VELOCITY AND VORTICITY, II
}

\author{
ELAINE COZZI
}

\begin{abstract}
We consider solutions to the two-dimensional incompressible Navier-Stokes and Euler equations for which velocity and vorticity are bounded in the plane. We show that for every $T>0$, the Navier-Stokes velocity converges in $L^{\infty}\left([0, T] ; L^{\infty}\left(\mathbb{R}^{2}\right)\right)$ as viscosity approaches 0 to the Euler velocity generated from the same initial data. This improves our earlier results to the effect that the vanishing viscosity limit holds on a sufficiently short time interval, or for all time under the assumption of decay of the velocity vector field at infinity.
\end{abstract}

\section{Introduction}

In this paper, we study the vanishing viscosity limit of solutions to the twodimensional incompressible Navier-Stokes equations. Recall that the Navier-Stokes equations modeling incompressible viscous fluid flow on $\mathbb{R}^{n}$ are given by

$$
\left\{\begin{array}{l}
\partial_{t} u_{v}+u_{v} \cdot \nabla u_{v}-v \Delta u_{v}=-\nabla p_{v}, \\
\operatorname{div} u_{v}=0 \\
\left.u_{v}\right|_{t=0}=u_{v}^{0} .
\end{array}\right.
$$

When $v=0$, the Navier-Stokes equations reduce to the Euler equations modeling incompressible inviscid fluid flow on $\mathbb{R}^{n}$ :

$$
\left\{\begin{array}{l}
\partial_{t} u+u \cdot \nabla u=-\nabla p \\
\operatorname{div} u=0 \\
\left.u\right|_{t=0}=u^{0}
\end{array}\right.
$$

There are a number of results addressing the vanishing viscosity limit of solutions of (NS) on $\mathbb{R}^{n}$ under various assumptions on the initial data (see, for example, [Constantin 1986; Masmoudi 2007; Kelliher 2004; Chemin 1996; Kato 1972; Swann 1971]). Here we focus our attention on solutions to (NS) and (E) in the plane with bounded velocity and vorticity which do not necessarily decay at infinity. We show that such solutions to (NS) converge to solutions of (E) with the same initial

MSC2010: 76D05.

Keywords: fluid mechanics, inviscid limit. 
data in the $L^{\infty}$-norm, where convergence is uniform over any finite time interval. This result builds upon and is a continuation of work in [Cozzi 2009; 2010]. For this reason, we will often refer to these articles for background information and useful estimates.

The existence and uniqueness of solutions to (NS) without any decay assumptions on the initial velocity is considered by Giga, Inui, and Matsui in [Giga et al. 1999]. The authors establish the short-time existence and uniqueness of mild solutions $v_{v}$ to (NS) in the space $C\left(\left[0, T_{0}\right] ; B U C\left(\mathbb{R}^{n}\right)\right)$ when initial velocity is in $B U C\left(\mathbb{R}^{n}\right)$ and $n \geq 2$. Here $B U C\left(\mathbb{R}^{n}\right)$ denotes the space of bounded uniformly continuous functions on $\mathbb{R}^{n}$. In [Giga et al. 2001], Giga, Matsui, and Sawada prove that when $n=2$, the unique solution can be extended globally in time. Existence and uniqueness of solutions to (E) with bounded velocity and vorticity with $n=2$ is due to Serfati [1995]. We briefly discuss these results in Section 2.

In this paper we prove that solutions $u_{v}$ to (NS) of [Giga et al. 2001] converge uniformly on $\mathbb{R}^{2}$ to Serfati solutions to (E) as viscosity approaches 0 , where convergence is uniform over any finite time interval (see Theorem 3). To establish the result, we apply Littlewood-Paley theory and Bony's paradifferential calculus [1981] and follow the general strategy of [Cozzi 2009; 2010]. Specifically, we consider low and high frequencies of the difference between the solutions to (NS) and (E) separately. We first show that for fixed $t$ and for any positive integer $n$,

$$
\left\|u_{v}(t)-u(t)\right\|_{L^{\infty}} \leq n\left\|u_{v}(t)-u(t)\right\|_{B_{\infty, \infty}^{0}}+2^{-n}\left\|\omega_{v}(t)-\omega(t)\right\|_{L^{\infty}}
$$

where $\omega_{v}=\operatorname{curl} u_{v}$ and $\omega=\operatorname{curl} u$. (See [Cozzi 2009] for a definition of the Besov space $B_{\infty, \infty}^{0}$.) Letting $n$ be a function of $v$ such that $n$ approaches $\infty$ as $v$ approaches 0 , we show that the right-hand side of (1.3) approaches 0 as $n$ approaches $\infty$. Since the second term on the right in (1.3) can be bounded above by $2^{-n}\left(\left\|\omega_{v}(t)\right\|_{L^{\infty}}+\|\omega(t)\|_{L^{\infty}}\right)$, we have essentially reduced the problem to proving that the vanishing viscosity limit holds in the $B_{\infty, \infty}^{0}$-norm. Since $L^{\infty}$ embeds continuously into $B_{\infty, \infty}^{0}$, we expect this problem to be easier than proving that the vanishing viscosity limit holds in the $L^{\infty}$-norm; however, we must establish a rate of convergence sufficiently fast to combat the growth of the factor of $n$ in front of the Besov norm.

Working in the Besov space $B_{\infty, \infty}^{0}$ has several advantages over working in $L^{\infty}$. Recall that for two-dimensional fluids we can express the Euler velocity gradient in terms of its vorticity by the relation $\nabla u=\nabla \nabla^{\perp} \Delta^{-1} \omega$. We can also express the Euler pressure in terms of velocity by the equality $p(t)=\sum_{i, j=1}^{2} R_{i} R_{j} u_{i} u_{j}(t)$, where $R_{i}$ denotes the Riesz operator (similar relations hold for the Navier-Stokes velocity, vorticity, and pressure). The main mathematical obstacle when studying solutions to fluid equations in $L^{\infty}$ is the lack of boundedness of the Calderon-Zygmund 
operators $\nabla \nabla^{\perp} \Delta^{-1}$ and $R_{j} R_{i}$ on $L^{\infty}$. However, if we let $\Delta_{j}$ denote the LittlewoodPaley operator which projects in frequency space onto an annulus with inner and outer radius of order $2^{j}$, then for any $j \geq 0, f \in \mathcal{S}^{\prime}$, and Calderon-Zygmund operator $A$, we have

$$
\left\|\Delta_{j} A f\right\|_{L^{\infty}} \leq\left\|\Delta_{j} f\right\|_{L^{\infty}} .
$$

Therefore, when proving estimates in the $B_{\infty, \infty}^{0}$-norm, we can localize the frequencies of (NS) and (E) by applying the Littlewood-Paley operator $\Delta_{j}$ to the equations. We can then estimate the difference $\Delta_{j}\left(u_{v}-u\right)$ in the $L^{\infty}$-norm using (1.4). The presence of the Littlewood-Paley operator thus facilitates estimates for velocity gradients and pressure terms.

In [Cozzi 2009] we proved that when $u, u_{v}, \omega$ and $\omega_{v}$ belong to $L_{\text {loc }}^{\infty}\left(\mathbb{R}^{+} ; L^{\infty}\left(\mathbb{R}^{2}\right)\right)$, there exists $T>0$ such that

$$
\left\|u_{v}-u\right\|_{L^{\infty}\left([0, T] ; L^{\infty}\left(\mathbb{R}^{2}\right)\right)} \rightarrow 0 \text { as } v \rightarrow 0 .
$$

To show (1.5), we reduced the problem to showing that the vanishing viscosity limit holds in the homogeneous $\dot{B}_{\infty, \infty}^{0}$-norm, but we were only able to show convergence in this norm for short time. In this paper, we show that (1.5) holds for every $T>0$ by showing that the vanishing viscosity limit holds in the inhomogeneous $B_{\infty, \infty}^{0}$-norm on any finite time interval $[0, T]$.

We remark that this improvement of our previous result is not a consequence of using the inhomogeneous norm in place of the homogeneous norm. In fact, we are able to prove the same convergence result regardless of which Besov norm we use (the proof using the inhomogeneous norm is cleaner). Rather, in this paper we are able to improve upon the results in [Cozzi 2009] because we change our approach when estimating the commutator resulting from an application of the Littlewood-Paley operator to the nonlinear terms in (NS) and (E). Our approach here is similar to those in [Vishik 1999; Bahouri and Chemin 1994; Taniuchi et al. 2010]. As a result of our methods, we are able to prove the estimate

$$
\left\|\left(u_{v}-u\right)(t)\right\|_{B_{\infty, \infty}^{0}} \leq C(T) 2^{-n \alpha}+\int_{0}^{t} C\left(2^{-p}+p\left\|\left(u_{v}-u\right)(s)\right\|_{B_{\infty, \infty}^{0}}\right)
$$

for any $p \in[2, \infty)$. By choosing $p$ as a logarithmic function of $\left\|u_{v}-u\right\|_{B_{\infty, \infty}^{0}}$, we are able to apply Osgood's lemma, yielding a rate of convergence. In [Cozzi 2009], our methods only allow us to prove an estimate similar to (1.6) with $n$ in place of $p$. Since $n$ is a function of viscosity, we must apply Gronwall's lemma and introduce a factor of $e^{n t}$ on the right hand side, which prevents us from proving that the inviscid limit holds on any finite time interval.

The paper is organized as follows. In Section 2, we review properties of nondecaying solutions to the fluid equations. In Section 3 and Section 4, we state and 
prove the main result; we devote Section 4 entirely to showing that the vanishing viscosity limit holds in the $B_{\infty, \infty}^{0}$-norm.

For background information on Littlewood-Paley theory, Bony's paraproduct decomposition, Besov spaces, and technical lemmas used throughout the paper, we refer the reader to Section 2 of [Cozzi 2009].

\section{Existence and uniqueness of nondecaying solutions to the fluid equations}

In this section, we briefly summarize what is known about nondecaying solutions to (NS) and (E). We begin with the mild solutions to (NS) established in [Giga et al. 1999]. By a mild solution to (NS), we mean a solution $u_{v}$ of the integral equation

$$
u_{v}(t, x)=e^{t v \Delta} u_{v}^{0}-\int_{0}^{t} e^{(t-s) v \Delta} \mathbf{P}\left(u_{v} \cdot \nabla u_{v}\right)(s) d s .
$$

In (2.1), $e^{\tau \nu \Delta}$ denotes convolution with the Gauss kernel; that is, for $f \in S^{\prime}$, $e^{\tau \nu \Delta} f=G_{\tau v} * f$, where $G_{\tau v}(x)=1 /(4 \pi \tau \nu) \exp \left(-|x|^{2} /(4 \tau \nu)\right)$. Also, $\mathbf{P}$ denotes the Helmholtz projection operator with $i, j$ component given by $\delta_{i j}+R_{i} R_{j}$, where $R_{l}=(-\Delta)^{-1 / 2} \partial_{l}$ is the Riesz operator. Giga, Inui, and Matsui proved the following result regarding mild solutions in $\mathbb{R}^{n}, n \geq 2$ :

Theorem 1 [Giga et al. 1999]. Let BUC denote the space of bounded uniformly continuous functions, and assume $u_{v}^{0}$ belongs to $B U C\left(\mathbb{R}^{n}\right)$ for fixed $n \geq 2$. There exists $T_{0}>0$ and a unique solution to $(2.1)$ in the space $C\left(\left[0, T_{0}\right] ; B U C\left(\mathbb{R}^{n}\right)\right)$ with initial data $u_{v}^{0}$. Moreover, if we assume div $u_{v}^{0}=0$, and if we define $p_{v}(t)=$ $\sum_{i, j=1}^{2} R_{i} R_{j} u_{v i} u_{v j}(t)$ for each $t \in\left[0, T_{0}\right]$, then $u_{v}$ belongs to $C^{\infty}\left(\left[0, T_{0}\right] \times \mathbb{R}^{n}\right)$ and solves $(\mathrm{NS})$.

Remark 2.2. For the main theorem of this paper, we assume that $u^{0}$ and $\omega^{0}$ are bounded on $\mathbb{R}^{2}$ and that $\operatorname{div} u^{0}=0$. These assumptions imply that $u^{0}$ belongs to $C^{\alpha}\left(\mathbb{R}^{2}\right)$ for every $\alpha<1$ and is therefore in $B U C\left(\mathbb{R}^{2}\right)$ (see, for example, Lemma 4 of [Cozzi 2009]).

Giga, Matsui, and Sawada [2001] showed that when $n=2$, the solution to (NS) established in Theorem 1 can be extended to a global-in-time smooth solution. Sawada and Taniuchi [2007] showed that if $u_{v}^{0}$ and $\omega_{v}^{0}$ belong to $L^{\infty}\left(\mathbb{R}^{2}\right)$, then the following exponential estimate holds:

$$
\left\|u_{v}(t)\right\|_{L^{\infty}} \leq C\left\|u_{v}^{0}\right\|_{L^{\infty}} e^{C t\left\|\omega_{v}^{0}\right\|_{L^{\infty}}} .
$$

For ideal incompressible fluids, we have the following result:

Theorem 2 [Serfati 1995]. Let $u^{0}$ and $\omega^{0}$ belong to $L^{\infty}\left(\mathbb{R}^{2}\right)$, and let $c \in \mathbb{R}$. For every $T>0$, there exists a unique solution $(u, p)$ to $(\mathrm{E})$ in the space

$$
L^{\infty}\left([0, T] ; L^{\infty}\left(\mathbb{R}^{2}\right)\right) \times L^{\infty}\left([0, T] ; C\left(\mathbb{R}^{2}\right)\right)
$$


with $\omega \in L^{\infty}\left([0, T] ; L^{\infty}\left(\mathbb{R}^{2}\right)\right), p(0)=c$, and with $p(t, x) /|x| \rightarrow 0$ as $|x| \rightarrow \infty$.

Serfati also proved an estimate analogous to (2.3) for his solutions:

$$
\|u(t)\|_{L^{\infty}} \leq C\left\|u^{0}\right\|_{L^{\infty}} e^{C_{1}\left\|\omega^{0}\right\|_{L^{\infty}} t} .
$$

Finally, we recall that we have a uniform bound in time on the $L^{\infty}$-norms of the vorticities corresponding to the solutions of (NS) and (E). For fixed $v \geq 0$, we have that

$$
\left\|\omega_{\nu}(t)\right\|_{L^{\infty}} \leq\left\|\omega_{\nu}^{0}\right\|_{L^{\infty}}
$$

for all $t \geq 0$. One can prove this bound by applying the maximum principle to the vorticity formulations of (NS) and (E). We refer the reader to Lemma 3.1 of [Sawada and Taniuchi 2007] for a detailed proof.

\section{Statement and proof of the main result}

We are now prepared to state the main theorem:

Theorem 3. Let $u_{v}$ be the unique solution to (NS) and $u$ the unique solution to (E), both with initial data $u^{0}$ and $\omega^{0}$ belonging to $L^{\infty}\left(\mathbb{R}^{2}\right)$, and with $p_{v}$ and $p$ satisfying the conditions of Theorems 1 and 2, respectively. Let $M$ be defined by (3.2) below and let $T>0$ be fixed. Then there exists a constant $C_{M, T}$, increasing with both $M$ and $T$, such that the following estimate holds for any fixed $\alpha \in(0,1)$ :

$$
\left\|u_{v}-u\right\|_{L^{\infty}\left([0, T] ; L^{\infty}\left(\mathbb{R}^{2}\right)\right)} \leq C_{M, T}\left(2-\log (\sqrt{v})^{\alpha e^{-C_{M, T}}}\right)(\sqrt{v})^{\alpha e^{-C_{M, T}}} .
$$

Proof. Throughout the proof of Theorem 3, we let $M$ denote a constant, dependent on $T$, which satisfies

$$
M \geq 1+\sup _{t \in[0, T]}\left(\left\|u_{v}(t)\right\|_{L^{\infty}}+\|u(t)\|_{L^{\infty}}+\left\|\omega_{\nu}(t)\right\|_{L^{\infty}}+\|\omega(t)\|_{L^{\infty}}\right) .
$$

We note that the value of $M$ will change throughout the proof but will always satisfy (3.2). The existence results in Section 2 imply that $M$ will be finite for any $T>0$.

Let $u$ be the unique Serfati solution to (E), and let $u_{v}$ be the unique solution to (NS) given by [Giga et al. 2001]. We fix $n$ to be a positive integer and we fix $T>0$. We will eventually choose $n=-\frac{1}{2} \log _{2} v$ so that as $v$ approaches $0, n$ approaches $\infty$. 
We begin with the following inequality:

$$
\begin{aligned}
\left\|u_{v}-u\right\|_{L^{\infty}\left([0, T] ; L^{\infty}\right)} & \leq \sum_{j=-1}^{n}\left\|\Delta_{j}\left(u_{v}-u\right)\right\|_{L^{\infty}\left([0, T] ; L^{\infty}\right)} \\
& +\sum_{j=n+1}^{\infty}\left\|\Delta_{j}\left(u_{v}-u\right)\right\|_{L^{\infty}\left([0, T] ; L^{\infty}\right)} .
\end{aligned}
$$

We can estimate the second term on the right-hand side of (3.3) using Bernstein's lemma and the estimate

$$
\left\|\Delta_{j} \nabla u\right\|_{L^{\infty}} \leq\left\|\Delta_{j} \omega\right\|_{L^{\infty}} \text { for } j \geq 0 .
$$

(Both (3.4) and Bernstein's lemma can be found in Section 2 of [Cozzi 2009].) We obtain the inequality

$$
\begin{aligned}
\sum_{j=n+1}^{\infty}\left\|\Delta_{j}\left(u_{v}-u\right)\right\|_{L^{\infty}\left([0, T] ; L^{\infty}\right)} & \leq \sum_{j=n+1}^{\infty} 2^{-j}\left\|\Delta_{j}\left(\nabla u_{v}-\nabla u\right)\right\|_{L^{\infty}\left([0, T] ; L^{\infty}\right)} \\
& \leq M 2^{-n}
\end{aligned}
$$

To estimate the first term on the right-hand side of (3.3), we use the definition of $B_{\infty, \infty}^{0}$ to observe that

$$
\sum_{j=-1}^{n}\left\|\Delta_{j}\left(u_{v}-u\right)\right\|_{L^{\infty}\left([0, T] ; L^{\infty}\right)} \leq C n\left\|u_{v}-u\right\|_{L^{\infty}\left([0, T] ; B_{\infty, \infty}^{0}\right)} .
$$

After substituting (3.6) and (3.5) into (3.3), we conclude that

$$
\left\|u_{v}-u\right\|_{L^{\infty}\left([0, T] ; L^{\infty}\right)} \leq C n\left\|u_{\nu}-u\right\|_{L^{\infty}\left([0, T] ; B_{\infty, \infty}^{0}\right)}+M 2^{-n}
$$

We must estimate the difference of $u_{v}$ and $u$ in the $B_{\infty, \infty}^{0}$-norm. We temporarily assume that the following estimate holds for all $\alpha \in(0,1)$ :

$$
\left\|u_{v}-u\right\|_{L^{\infty}\left([0, T] ; B_{\infty, \infty}^{0}\right)} \leq C_{M, T}\left(2-\log 2^{-n \alpha e^{-C_{M, T}}}\right) 2^{-n \alpha e^{-C_{M, T}}} .
$$

Assuming that (3.8) holds, we see from (3.7) and (3.8) that

$$
\left\|u_{v}-u\right\|_{L^{\infty}\left([0, T] ; L^{\infty}\right)} \leq C_{M, T}\left(2-\log 2^{-n \alpha e^{-C_{M, T}}}\right) 2^{-n \alpha e^{-C_{M, T}}} .
$$

The estimate (3.1) follows after setting $v=2^{-2 n}$. Therefore, to complete the proof of Theorem 3, it remains to prove (3.8). 


\section{Proof of (3.8)}

Let $u_{n}=S_{n} u, \omega_{n}=S_{n} \omega(u), \bar{u}_{n}=u_{v}-u_{n}$, and $\bar{\omega}_{n}=\omega_{v}-\omega_{n}$. Throughout most of the proof of (3.8), the time $t$ is fixed and suppressed in the calculations.

Fix $p \in(1, \infty)$ (to be chosen later). We apply Bernstein's lemma and (3.4) to establish the estimate

$$
\begin{gathered}
\left\|u_{v}-u\right\|_{B_{\infty, \infty}^{0}} \leq \sup _{-1 \leq l \leq 2}\left\|\Delta_{l}\left(u_{v}-u\right)\right\|_{L^{\infty}}+\sup _{3 \leq l \leq p} 2^{-l}\left\|\Delta_{l}\left(\omega_{v}-\omega\right)\right\|_{L^{\infty}} \\
+\sup _{l>p} 2^{-l}\left\|\Delta_{l}\left(\omega_{v}-\omega\right)\right\|_{L^{\infty}} .
\end{gathered}
$$

The separation of frequencies at $l=2$ will simplify estimates in what follows.

We will first consider the difference $\sup _{3 \leq l \leq p} 2^{-l}\left\|\Delta_{l}\left(\omega_{v}-\omega\right)\right\|_{L^{\infty}}$. We will eventually need to estimate the viscosity term $v\|\Delta \omega\|_{L^{\infty}}$. To facilitate this estimate, we smooth out the Euler vorticity and write

$$
\begin{aligned}
\sup _{3 \leq l \leq p} 2^{-l}\left\|\Delta_{l}\left(\omega_{v}-\omega\right)\right\|_{L^{\infty}} & \leq \sup _{3 \leq l \leq p} 2^{-l}\left\|\Delta_{l} \bar{\omega}_{n}\right\|_{L^{\infty}}+\sup _{3 \leq l \leq p} 2^{-l}\left\|\Delta_{l}\left(\omega_{n}-\omega\right)\right\|_{L^{\infty}} \\
& \leq \sup _{3 \leq l \leq p} 2^{-l}\left\|\Delta_{l} \bar{\omega}_{n}\right\|_{L^{\infty}}+\sup _{l \geq n} 2^{-l}\left\|\Delta_{l}\left(\omega_{n}-\omega\right)\right\|_{L^{\infty}} \\
& \leq \sup _{3 \leq l \leq p} 2^{-l}\left\|\Delta_{l} \bar{\omega}_{n}\right\|_{L^{\infty}}+M 2^{-n},
\end{aligned}
$$

where we used properties of the Fourier support of $\omega_{n}$ to get the second inequality. We now estimate $\sup _{3 \leq l \leq p} 2^{-l}\left\|\Delta_{l} \bar{\omega}_{n}\right\|_{L^{\infty}}$. We note that $\omega_{\nu}$ and $\omega_{n}$ satisfy

$$
\partial_{t} \omega_{v}+u_{v} \cdot \nabla \omega_{v}-v \Delta \omega_{v}=0
$$

and

$$
\partial_{t} \omega_{n}+u_{n} \cdot \nabla \omega_{n}=\nabla \cdot \tau_{n}(u, \omega),
$$

where $\tau_{n}(u, \omega)=\left(u-u_{n}\right)\left(\omega-\omega_{n}\right)-r_{n}(u, \omega)$ and

$$
r_{n}(u, \omega)=\int \check{\psi}_{0}(y)\left(u\left(x-2^{-n} y\right)-u(x)\right)\left(\omega\left(x-2^{-n} y\right)-\omega(x)\right) d y .
$$

Here $\psi_{0}$ denotes the Fourier multiplier associated with the Littlewood-Paley operator $\Delta_{-1}$. Equation (4.4) was utilized by Constantin and $\mathrm{Wu}$ [1996] and by Constantin, $\mathrm{E}$, and Titi in a proof of Onsager's conjecture in [Constantin et al. 1994]. We subtract (4.4) from (4.3) and, for fixed $l$, we apply the Littlewood-Paley operator $\Delta_{l}$ to the difference of the two equations. After adding $\left(S_{l-2} u_{v}\right) \cdot \nabla \Delta_{l} \bar{\omega}_{n}$ to both 
sides of the resulting equation, we obtain

$$
\begin{aligned}
\partial_{t} \Delta_{l} \bar{\omega}_{n}+\left(S_{l-2} u_{v}\right) & \cdot \nabla \Delta_{l} \bar{\omega}_{n}-v \Delta_{l} \Delta_{l} \bar{\omega}_{n} \\
=\left(S_{l-2} u_{v}\right) & \cdot \nabla \Delta_{l} \bar{\omega}_{n}-\Delta_{l}\left(u_{v} \cdot \nabla \bar{\omega}_{n}\right) \\
& \quad-\Delta_{l}\left(\bar{u}_{n} \cdot \nabla \omega_{n}\right)+v \Delta_{l} \omega_{n}-\Delta_{l} \nabla \cdot \tau_{n}(u, \omega) .
\end{aligned}
$$

Borrowing notation from [Taniuchi et al. 2010], we define

$$
I^{l, k}=\left(S_{l-2} u_{\nu}^{k}\right) \partial_{k} \Delta_{l} \bar{\omega}_{n}-\partial_{k} \Delta_{l}\left(u_{\nu}^{k} \bar{\omega}_{n}\right) \quad \text { and } \quad J^{l, k}=-\partial_{k} \Delta_{l}\left(\bar{u}_{n}^{k} \omega_{n}\right) .
$$

From (4.5), we see that

$$
\begin{aligned}
\partial_{t} \Delta_{l} \bar{\omega}_{n}+\left(S_{l-2} u_{v}\right) \cdot \nabla \Delta_{l} \bar{\omega}_{n} & -v \Delta_{l} \bar{\omega}_{n} \\
& =\sum_{k=1}^{2}\left(I^{l, k}+J^{l, k}\right)+v \Delta_{l} \omega_{n}-\Delta_{l} \nabla \cdot \tau_{n}(u, \omega) .
\end{aligned}
$$

Since $S_{l-2} u_{v}$ belongs to $L_{\text {loc }}^{1}\left(\mathbb{R}^{+} ; \operatorname{Lip}\left(\mathbb{R}^{2}\right)\right)$ and is divergence-free, we can apply the following lemma for the transport diffusion equation from [Hmidi 2005].

Lemma 4. Let $p \in[1, \infty]$, and let $u$ be a divergence-free vector field belonging to $L_{\text {loc }}^{1}\left(\mathbb{R}^{+} ; \operatorname{Lip}\left(\mathbb{R}^{d}\right)\right)$. Moreover, assume the function $f$ belongs to $L_{\text {loc }}^{1}\left(\mathbb{R}^{+} ; L^{p}\left(\mathbb{R}^{d}\right)\right)$ and the function $a^{0}$ belongs to $L^{p}\left(\mathbb{R}^{d}\right)$. Then any solution a to the problem

$$
\left\{\begin{array}{l}
\partial_{t} a+u \cdot \nabla a-v \Delta a=f \\
\left.a\right|_{t=0}=a^{0}
\end{array}\right.
$$

satisfies the estimate

$$
\|a(t)\|_{L^{p}} \leq\left\|a^{0}\right\|_{L^{p}}+\int_{0}^{t}\|f(s)\|_{L^{p}} d s .
$$

An application of Lemma 4 to (4.7) yields

$$
\begin{array}{r}
\left\|\Delta_{l} \bar{\omega}_{n}(t)\right\|_{L^{\infty}} \leq\left\|\Delta_{l} \bar{\omega}_{n}(0)\right\|_{L^{\infty}}+\int_{0}^{t}\left(\sum_{k=1}^{2}\left(\left\|I^{l, k}(s)\right\|_{L^{\infty}}+\left\|J^{l, k}(s)\right\|_{L^{\infty}}\right)\right) d s \\
+\int_{0}^{t}\left(v\left\|\Delta \Delta_{l} \omega_{n}(s)\right\|_{L^{\infty}}+\left\|\Delta_{l} \nabla \cdot \tau_{n}(u, \omega)(s)\right\|_{L^{\infty}}\right) d s .
\end{array}
$$

Our goal is to establish an upper bound for $\sup _{3 \leq l \leq p} 2^{-l}\left\|\Delta_{l} \bar{\omega}_{n}(t)\right\|_{L^{\infty}}$. In what follows, we will estimate each term on the right-hand side of (4.8), multiply by $2^{-l}$, and take the supremum over $l$ satisfying $3 \leq l \leq p$. Estimates for the last two terms on the right-hand side of (4.8) follow from work in [Cozzi 2009]. Indeed, in that paper we used boundedness of the Euler vorticity and membership of the Euler 
velocity in $C^{\alpha}\left(\mathbb{R}^{2}\right)$ for any $\alpha \in(0,1)$ to show that for such $\alpha$,

$$
\sup _{l \geq 0} 2^{-l}\left\|\Delta_{l} \nabla \cdot \tau_{n}(u, \omega)\right\|_{L^{\infty}} \leq\left\|\nabla \cdot \tau_{n}(u, \omega)\right\|_{L^{\infty}} \leq M 2^{-n \alpha} .
$$

We also showed there, using Bernstein's lemma and properties of the Fourier support of $\omega_{n}$, that

$$
\sup _{l \geq 0} 2^{-l} v\left\|\Delta_{l} \Delta \omega_{n}\right\|_{L^{\infty}} \leq 2^{n} v\left\|\omega_{n}\right\|_{L^{\infty}} \leq M 2^{-n},
$$

where we set $v=2^{-2 n}$. To estimate the initial data, we used the Fourier support of $\omega_{n}^{0}=S_{n} \omega^{0}$ to write

$$
\sup _{3 \leq l \leq p} 2^{-l}\left\|\Delta_{l} \bar{\omega}_{n}(0)\right\|_{L^{\infty}} \leq \sup _{l \geq n} 2^{-l}\left\|\Delta_{l} \bar{\omega}_{n}(0)\right\|_{L^{\infty}} \leq M 2^{-n} .
$$

Multiplying (4.8) by $2^{-l}$, taking the supremum of (4.8) over $l$ satisfying $3 \leq l \leq p$, and applying the estimates (4.9), (4.10), and (4.11) gives

$$
\begin{aligned}
& \sup _{3 \leq l \leq p} 2^{-l}\left\|\Delta_{l} \bar{\omega}_{n}(t)\right\|_{L^{\infty}} \\
& \quad \leq M(t+1) 2^{-n \alpha}+\sup _{3 \leq l \leq p} 2^{-l} \int_{0}^{t}\left(\sum_{k=1}^{2}\left(\left\|I^{l, k}(s)\right\|_{L^{\infty}}+\left\|J^{l, k}(s)\right\|_{L^{\infty}}\right)\right) d s .
\end{aligned}
$$

It remains to estimate $I^{l, k}$ and $J^{l, k}$. We begin with $J^{l, k}$. We again borrow notation from [Taniuchi et al. 2010] and use Bony's paraproduct decomposition to write

$$
\begin{gathered}
J^{l, k}=-\partial_{k} \Delta_{l} \sum_{\substack{|j-l| \leq 3 \\
j \geq 1}} S_{j-2} \bar{u}_{n}^{k} \Delta_{j} \omega_{n} \\
-\partial_{k} \Delta_{l} \sum_{\substack{|j-l| \leq 3 \\
j \geq 1}} \Delta_{j} \bar{u}_{n}^{k} S_{j-2} \omega_{n} \\
-\partial_{k} \Delta_{l} \sum_{\substack{\left|j-j^{\prime}\right| \leq 1 \\
\max \left\{j, j^{\prime}\right\} \geq l-3}} \Delta_{j} \bar{u}_{n}^{k} \Delta_{j^{\prime}} \omega_{n} \\
=J_{1}^{l, k}+J_{2}^{l, k}+J_{3}^{l, k} .
\end{gathered}
$$

We estimate $J_{1}^{l, k}$. Several applications of Bernstein's lemma give

$$
\begin{aligned}
\left\|J_{1}^{l, k}\right\|_{L^{\infty}} & \leq 2^{l} \sum_{\substack{|j-l| \leq 3 \\
j \geq 1}}\left\|S_{j-2} \bar{u}_{n}\right\|_{L^{\infty}}\left\|\Delta_{j} \omega_{n}\right\|_{L^{\infty}} \\
& \leq 2^{l} \sum_{\substack{|j-l| \leq 3 \\
j \geq 1}}\left\|\Delta_{j} \omega\right\|_{L^{\infty}} \sum_{k \leq j-2}\left\|\Delta_{k} \bar{u}_{n}\right\|_{L^{\infty}} .
\end{aligned}
$$


Multiplying by $2^{-l}$ and taking the supremum over $l$ satisfying $3 \leq l \leq p$, we conclude that

$$
\sup _{3 \leq l \leq p} 2^{-l}\left\|J_{1}^{l, k}\right\|_{L^{\infty}} \leq M p\left\|\bar{u}_{n}\right\|_{B_{\infty, \infty}^{0}} .
$$

We now estimate $J_{2}^{l, k}$. We write

$$
\begin{aligned}
\left\|J_{2}^{l, k}\right\|_{L^{\infty}} & \leq 2^{l} \sum_{\substack{|j-l| \leq 3 \\
j \geq 1}}\left\|\Delta_{l}\left(\Delta_{j} \bar{u}_{n} S_{j-2} \omega_{n}\right)\right\|_{L^{\infty}} \\
& \leq 2^{l} \sum_{\substack{|j-l| \leq 3 \\
j \geq 1}}\left\|\Delta_{j} \bar{u}_{n}\right\|_{L^{\infty}}\left\|S_{j-2} \omega_{n}\right\|_{L^{\infty}}
\end{aligned}
$$

so that

$$
\sup _{3 \leq l \leq p} 2^{-l}\left\|J_{2}^{l, k}\right\|_{L^{\infty}} \leq M\left\|\bar{u}_{n}\right\|_{B_{\infty, \infty}^{0}}
$$

To estimate $J_{3}^{l, k}$, we use properties of Littlewood-Paley operators to observe that

$$
\begin{aligned}
\left\|J_{3}^{l, k}\right\|_{L^{\infty}} & \leq 2^{l} \sum_{\substack{\left|j-j^{\prime}\right| \leq 1 \\
\max \left\{j, j^{\prime}\right\} \geq l-3}}\left\|\Delta_{j} \bar{u}_{n}\right\|_{L^{\infty}}\left\|\Delta_{j^{\prime}} \omega_{n}\right\|_{L^{\infty}} \\
& \leq C 2^{l} \sum_{j \geq l-3}\left\|\Delta_{j} \bar{u}_{n}\right\|_{L^{\infty}}\left\|\Delta_{j} \omega_{n}\right\|_{L^{\infty}} \leq C 2^{l}\|\omega\|_{L^{\infty}}\left\|\bar{u}_{n}\right\|_{B_{\infty, 1}^{0}} .
\end{aligned}
$$

We estimate the $B_{\infty, 1}^{0}$-norm of $\bar{u}_{n}$ as follows: We bound the low frequencies using the definition of $B_{\infty, \infty}^{0}$, and we estimate the high frequencies using Bernstein's lemma, (3.4), and boundedness of vorticity. We have the series of estimates

(4.19) $\left\|\bar{u}_{n}\right\|_{B_{\infty, 1}^{0}} \leq \sum_{j=-1}^{p}\left\|\Delta_{j} \bar{u}_{n}\right\|_{L^{\infty}}+\sum_{j>p} 2^{-j}\left\|\Delta_{j} \bar{\omega}_{n}\right\|_{L^{\infty}} \leq C p\left\|\bar{u}_{n}\right\|_{B_{\infty, \infty}^{0}}+M 2^{-p}$.

Substituting this estimate into (4.18), multiplying by $2^{-l}$ and taking the supremum over $l$ between 3 and $p$ yields the estimate

$$
\sup _{3 \leq l \leq p} 2^{-l}\left\|J_{3}^{l, k}\right\|_{L^{\infty}} \leq M\left(2^{-p}+p\left\|\bar{u}_{n}\right\|_{B_{\infty, \infty}^{0}}\right) .
$$

Combining the estimates for (4.15), (4.17), and (4.20), we conclude that

$$
\sup _{3 \leq l \leq p} 2^{-l} \sum_{k=1}^{2}\left\|J^{l, k}\right\|_{L^{\infty}} \leq M\left(2^{-p}+p\left\|\bar{u}_{n}\right\|_{B_{\infty, \infty}^{0}}\right) .
$$


We now estimate $I^{l, k}$ for $l$ satisfying $3 \leq l \leq p$. We apply Theorem 6.1 of [Vishik 1999] to write

$$
\begin{aligned}
\sum_{k=1}^{2}\left\|I^{l, k}\right\|_{L^{\infty}} \leq & C \sum_{|j-l| \leq 3}\left\|S_{j-2} \nabla \bar{\omega}_{n}\right\|_{L^{\infty}}\left\|\Delta_{j} u_{v}\right\|_{L^{\infty}} \\
& +\sum_{|j-l| \leq 3}\left\|S_{j-2} \nabla u_{v}\right\|_{L^{\infty}}\left\|\Delta_{j} \bar{\omega}_{n}\right\|_{L^{\infty}} \\
& +C 2^{l} \sum_{\substack{j \geq l-3 \\
\left|j-j^{\prime}\right| \leq 1}} 2^{-j}\left\|\Delta_{j} \nabla u_{v}\right\|_{L^{\infty}}\left\|\Delta_{j^{\prime}} \bar{\omega}_{n}\right\|_{L^{\infty}} \\
= & X_{1}^{l}+X_{2}^{l}+X_{3}^{l} .
\end{aligned}
$$

To estimate $X_{1}^{l}$, keeping in mind that $l \geq 3$, we use Bernstein's lemma and (3.4) to write

$$
\sum_{|j-l| \leq 3}\left\|S_{j-2} \nabla \bar{\omega}_{n}\right\|_{L^{\infty}}\left\|\Delta_{j} u_{v}\right\|_{L^{\infty}} \leq C 2^{l} \sum_{|j-l| \leq 3}\left\|S_{j-2} \bar{u}_{n}\right\|_{L^{\infty}}\left\|\Delta_{j} \omega_{v}\right\|_{L^{\infty}}
$$

The remainder of the estimate for $X_{1}^{l}$ is identical to that for $J_{1}^{l, k}$. Multiplying by $2^{-l}$ and taking the supremum over $l$ between 3 and $p$, we conclude that

$$
\sup _{3 \leq l \leq p} 2^{-l} X_{1}^{l} \leq M p\left\|\bar{u}_{n}\right\|_{B_{\infty, \infty}^{0}}
$$

To estimate $X_{2}^{l}$ for $3 \leq l \leq p$, we again apply Bernstein's lemma and (3.4) to write

$$
\begin{aligned}
X_{2}^{l} & =\sum_{|j-l| \leq 3}\left\|S_{j-2} \nabla u_{v}\right\|_{L^{\infty}}\left\|\Delta_{j} \bar{\omega}_{n}\right\|_{L^{\infty}} \\
& \leq C 2^{l} \sum_{|j-l| \leq 3}\left(\left\|u_{\nu}\right\|_{L^{\infty}}+(j-1)\left\|\omega_{\nu}\right\|_{L^{\infty}}\right)\left\|\Delta_{j} \bar{u}_{n}\right\|_{L^{\infty}} \\
& \leq M l 2^{l} \sum_{|j-l| \leq 3}\left\|\Delta_{j} \bar{u}_{n}\right\|_{L^{\infty}} .
\end{aligned}
$$

To get the first inequality above, we bounded the term $\left\|S_{j-2} \nabla u_{v}\right\|_{L^{\infty}}$ above by the sum resulting from the $S_{j-2}$ operator. We then applied (3.4). After multiplying (4.23) by $2^{-l}$ and taking the supremum over $l$ satisfying $3 \leq l \leq p$, we find that

$$
\sup _{3 \leq l \leq p} 2^{-l} X_{2}^{l} \leq M p\left\|\bar{u}_{n}\right\|_{B_{\infty, \infty}^{0}}
$$


The estimate for $X_{3}^{l}$ is similar to that for $J_{3}^{l, k}$. For $l$ satisfying $3 \leq l \leq p$, we write

$$
\begin{aligned}
X_{3}^{l} & =C 2^{l} \sum_{\substack{j \geq l-3 \\
\left|j-j^{\prime}\right| \leq 1}} 2^{-j}\left\|\Delta_{j} \nabla u_{v}\right\|_{L^{\infty}}\left\|\Delta_{j^{\prime}} \bar{\omega}_{n}\right\|_{L^{\infty}} \\
& \leq C 2^{l} \sum_{j \geq l-3}\left\|\Delta_{j} \omega_{\nu}\right\|_{L^{\infty}}\left\|\Delta_{j} \bar{u}_{n}\right\|_{L^{\infty}},
\end{aligned}
$$

where we used Bernstein's lemma and (3.4) to get the last inequality. We now use the same argument as in (4.18) and (4.19) to conclude that

$$
\sup _{3 \leq l \leq p} 2^{-l} X_{3}^{l} \leq M\left(2^{-p}+p\left\|\bar{u}_{n}\right\|_{B_{\infty, \infty}^{0}}\right) .
$$

Combining the above estimates for $X_{1}^{l}, X_{2}^{l}$, and $X_{3}^{l}$, we have

$$
\sup _{3 \leq l \leq p} 2^{-l} \sum_{k=1}^{2}\left\|I^{l, k}\right\|_{L^{\infty}} \leq M\left(2^{-p}+p\left\|\bar{u}_{n}\right\|_{B_{\infty, \infty}^{0}}\right) .
$$

Applying the estimates (4.21) and (4.27) to (4.12), we conclude that

$$
\text { (4.28) } \sup _{3 \leq l \leq p} 2^{-l}\left\|\Delta_{l} \bar{\omega}_{n}(t)\right\|_{L^{\infty}} \leq C(t+1) 2^{-n \alpha}+M \int_{0}^{t}\left(2^{-p}+p\|W(s)\|_{\dot{B}_{\infty, \infty}^{0}}\right) d s
$$

for any $\alpha \in(0,1)$. We substitute (4.28) into (4.2). This gives

$$
\begin{aligned}
\sup _{3 \leq l \leq p} 2^{-l}\left\|\Delta_{l}\left(\omega_{v}-\omega\right)(t)\right\|_{L^{\infty}} \leq & C(t+1) 2^{-n \alpha} \\
& +M \int_{0}^{t}\left(2^{-p}+p\left\|\bar{u}_{n}(s)\right\|_{\dot{B}_{\infty, \infty}^{0}}\right) d s .
\end{aligned}
$$

Inspection of (4.1) reveals that we must still estimate $\sup _{-1<l<2}\left\|\Delta_{l}\left(u_{v}-u\right)(t)\right\|_{L^{\infty}}$ and $\sup _{l>p} 2^{-l}\left\|\Delta_{l}\left(\omega_{\nu}-\omega\right)(t)\right\|_{L^{\infty}}$. These two terms are more straightforward. We estimate the term $\sup _{l>p} 2^{-l}\left\|\Delta_{l}\left(\omega_{\nu}-\omega\right)(t)\right\|_{L^{\infty}}$ by observing that

$$
\sup _{l>p} 2^{-l}\left\|\Delta_{l}\left(\omega_{\nu}-\omega\right)(t)\right\|_{L^{\infty}} \leq M 2^{-p} .
$$

To estimate $\sup _{-1<l<2}\left\|\Delta_{l}\left(u_{v}-u\right)(t)\right\|_{L^{\infty}}$, we use the velocity formulation. Setting $\bar{p}=p_{v}-p$ and $\bar{u}=u_{v}-u$, we subtract (E) from (NS). This gives

$$
\partial_{t} \bar{u}+u_{v} \cdot \nabla \bar{u}+\bar{u} \cdot \nabla u-v \Delta \bar{u}=-\nabla \bar{p}+v \Delta u_{v} .
$$

We apply $\Delta_{l}$ to (4.31) for $-1 \leq l \leq 2$. This gives

$$
\begin{aligned}
\partial_{t} \Delta_{l} \bar{u}+\left(\Delta_{l} u_{v}\right) \cdot \nabla \Delta_{l} \bar{u}-v \Delta_{l} \Delta \bar{u}= & \left(\Delta_{l} u_{v}\right) \cdot \nabla \Delta_{l} \bar{u}-\Delta_{l}\left(u_{v} \cdot \nabla \bar{u}\right) \\
& -\Delta_{l}(\bar{u} \cdot \nabla u)-\Delta_{l} \nabla \bar{p}+v \Delta_{l} \Delta u_{v} .
\end{aligned}
$$


Again by Lemma 4, we have

$$
\begin{aligned}
& \left\|\Delta_{l} \bar{u}(t)\right\|_{L^{\infty}} \\
& \quad \leq \int_{0}^{t}\left(\left\|\left(\Delta_{l} u_{v} \cdot \nabla \Delta_{l} \bar{u}\right)(s)\right\|_{L^{\infty}}+\left\|\Delta_{l}\left(u_{v} \cdot \nabla \bar{u}\right)(s)\right\|_{L^{\infty}}\right. \\
& \left.\quad+\left\|\Delta_{l}(\bar{u} \cdot \nabla u)(s)\right\|_{L^{\infty}}+\left\|\Delta_{l} \nabla \bar{p}(s)\right\|_{L^{\infty}}+v\left\|\Delta_{l} \Delta u_{v}(s)\right\|_{L^{\infty}}\right) d s .
\end{aligned}
$$

We have the following straightforward estimates, all which follow from Bernstein's lemma and the divergence-free property of the velocity:

$$
\begin{aligned}
& \left\|\left(\Delta_{l} u_{v}\right) \cdot \nabla \Delta_{l} \bar{u}\right\|_{L^{\infty}} \leq C\left\|u_{v}\right\|_{L^{\infty}} 2^{l}\left\|\Delta_{l} \bar{u}\right\|_{L^{\infty}} \leq M 2^{l}\|\bar{u}\|_{L^{\infty}}, \\
& \left\|\Delta_{l}\left(u_{v} \cdot \nabla \bar{u}\right)\right\|_{L^{\infty}} \leq C 2^{l}\left\|u_{v}\right\|_{L^{\infty}}\|\bar{u}\|_{L^{\infty}} \leq M 2^{l}\|\bar{u}\|_{L^{\infty}}, \\
& \left\|\Delta_{l}(\bar{u} \cdot \nabla u)\right\|_{L^{\infty}} \leq 2^{l}\|\bar{u}\|_{L^{\infty}}\|u\|_{L^{\infty}} \leq M 2^{l}\|\bar{u}\|_{L^{\infty}}, \\
& v\left\|\Delta_{l} \Delta u_{v}\right\|_{L^{\infty}} \leq C \nu 2^{2 l}\left\|u_{v}\right\|_{L^{\infty}} \leq M v 2^{2 l} .
\end{aligned}
$$

To estimate the pressure, we follow an argument in [Taniuchi et al. 2010]. For $0 \leq l \leq 2$, if $\varphi_{l}$ is the Fourier multiplier associated with $\Delta_{l}$, then

$$
\begin{aligned}
\left\|\Delta_{l} \nabla \bar{p}\right\|_{L^{\infty}} & =\left\|\sum_{i, i^{\prime}=1}^{2} R_{i} R_{i^{\prime}} \nabla \Delta_{l}\left(\bar{u}^{i} u^{i^{\prime}}+u_{v}^{i} \bar{u}^{i^{\prime}}\right)\right\|_{L^{\infty}} \\
& \leq\left\|R_{i} R_{i^{\prime}} \nabla \check{\varphi}_{l}\right\|_{L^{1}}\left\|\bar{u}^{i} u^{i^{\prime}}+u_{\nu}^{i} \bar{u}^{i^{\prime}}\right\|_{L^{\infty}} \leq M 2^{l}\|\bar{u}\|_{L^{\infty}},
\end{aligned}
$$

where we applied the estimates $\left\|R_{i} R_{i^{\prime}} \nabla \check{\varphi}_{l}\right\|_{L^{1}} \leq\left\|R_{i} R_{i^{\prime}} \nabla \check{\varphi}_{l}\right\|_{\mathcal{H}^{1}} \leq\left\|\nabla \check{\varphi}_{l}\right\|_{\mathcal{H}^{1}} \leq C 2^{l}$ to get the last inequality. For the case $l=-1$, we apply the same series of estimates as in (4.35) with $\check{\psi}_{0}$ in place of $\check{\varphi}_{l}$.

Substituting the estimates (4.34) and (4.35) into (4.33) and taking the supremum over $-1 \leq l \leq 2$ yields

$$
\sup _{-1 \leq l \leq 2}\left\|\Delta_{l} \bar{u}(t)\right\|_{L^{\infty}} \leq M \int_{0}^{t}\left(\|\bar{u}\|_{L^{\infty}}+2^{-2 n}\right),
$$

where we used the equality $v=2^{-2 n}$. We now apply the embedding $B_{\infty, 1}^{0} \hookrightarrow L^{\infty}$, along with (4.19), to conclude that

$$
\sup _{-1 \leq l \leq 2}\left\|\Delta_{l} \bar{u}(t)\right\|_{L^{\infty}} \leq M t 2^{-2 n}+M \int_{0}^{t}\left(p\|\bar{u}(s)\|_{B_{\infty, \infty}^{0}}+2^{-p}\right) d s .
$$

We substitute the estimates (4.37), (4.29), and (4.30) into (4.1). We conclude that

$$
\begin{aligned}
\sup _{l \geq-1}\left\|\Delta_{l} \bar{u}(t)\right\|_{L^{\infty}} \leq & M(T+1) 2^{-n \alpha}+M 2^{-p} \\
& +\int_{0}^{t} M\left(2^{-p}+p\|\bar{u}(s)\|_{B_{\infty, \infty}^{0}}\right) d s .
\end{aligned}
$$


To complete the proof of (3.8), we will apply Osgood's lemma to (4.38). We first note that by the embedding $L^{\infty} \hookrightarrow B_{\infty, \infty}^{0}$,

$$
\|\bar{u}(t)\|_{B_{\infty, \infty}^{0}} \leq\|\bar{u}(t)\|_{L^{\infty}} \leq\left\|u_{v}(t)\right\|_{L^{\infty}}+\|u(t)\|_{L^{\infty}} \leq M
$$

for all $t \in[0, T]$. For each $t \in[0, T]$, set

$$
\delta(t)=\frac{\int_{0}^{t}\|\bar{u}(s)\|_{B_{\infty, \infty}^{0}} d s}{M T} \leq 1,
$$

and set $p=2-\log \delta(t)$. Then (4.38) reduces to

(4.40) $\|\bar{u}(t)\|_{B_{\infty, \infty}^{0}} \leq M(T+1) 2^{-n \alpha}+M(T+1) \delta(t)+M^{2} T\left(2-\log _{2} \delta(t)\right) \delta(t)$.

Integrating both sides over $[0, t]$ and dividing both sides by $M T$ yields the inequality

$$
\delta(t) \leq(T+1) 2^{-n \alpha}+\left(\frac{T+1}{T}+M\right) \int_{0}^{t}\left(2-\log _{2} \delta(s)\right) \delta(s) d s .
$$

We are now in a position to use Osgood's lemma (see [Chemin and Lerner 1995]):

Lemma 5 (Osgood's lemma). Let $\rho$ be a measurable positive function, let $\gamma$ be a locally integrable positive function, and let $\mu$ be a continuous increasing function. Assume that for some number $\beta>0$, the function $\rho$ satisfies

$$
\rho(t) \leq \beta+\int_{t_{0}}^{t} \gamma(s) \mu(\rho(s)) d s .
$$

Then $-\phi(\rho(t))+\phi(\beta) \leq \int_{t_{0}}^{t} \gamma(s) d s$, where $\phi(x)=\int_{x}^{1} \frac{1}{\mu(r)} d r$.

We set $\mu(r)=r(2-\log r), \quad \rho(t)=\delta(t), \quad \beta=(T+1) 2^{-n \alpha}$, and

$$
\gamma(t)=\frac{T+1}{T}+M:=C_{0}(M, T),
$$

and we apply Osgood's lemma to obtain, for any $t \leq T$,

$$
-\log (2-\log \delta(t))+\log \left(2-\log \left((T+1) 2^{-n \alpha}\right)\right) \leq C_{0}(M, T) t .
$$

Taking the exponential twice gives

$$
\delta(t) \leq e^{2-2 e^{-C_{0}(M, T) t}}\left((T+1) 2^{-n \alpha}\right)^{e^{-C_{0}(M, T) t}} .
$$

The inequality (3.8) follows after substituting (4.42) into (4.40) and letting $v=2^{-2 n}$.

\section{Acknowledgement}

This work was supported by the National Science Foundation under grant DMS1049698. 


\section{References}

[Bahouri and Chemin 1994] H. Bahouri and J.-Y. Chemin, "Équations de transport relatives á des champs de vecteurs non-lipschitziens et mécanique des fluides", Arch. Rational Mech. Anal. 127:2 (1994), 159-181. MR 95g:35164 Zbl 0821.76012

[Bony 1981] J.-M. Bony, "Calcul symbolique et propagation des singularités pour les équations aux dérivées partielles non linéaires", Ann. Sci. École Norm. Sup. (4) 14:2 (1981), 209-246. MR 84h:35177 Zbl 0495.35024

[Chemin 1996] J.-Y. Chemin, "A remark on the inviscid limit for two-dimensional incompressible fluids", Comm. Partial Differential Equations 21:11-12 (1996), 1771-1779. MR 98c:35127 Zbl 0876.35087

[Chemin and Lerner 1995] J.-Y. Chemin and N. Lerner, "Flot de champs de vecteurs non lipschitziens et équations de Navier-Stokes", J. Differential Equations 121:2 (1995), 314-328. MR 96h:35153 Zbl 0878.35089

[Constantin 1986] P. Constantin, "Note on loss of regularity for solutions of the 3-D incompressible Euler and related equations", Comm. Math. Phys. 104:2 (1986), 311-326. MR 87f:35200 Zbl 0655.76041

[Constantin and $\mathrm{Wu}$ 1996] P. Constantin and J. Wu, "The inviscid limit for non-smooth vorticity", Indiana Univ. Math. J. 45:1 (1996), 67-81. MR 97g:35129 Zbl 0859.76015

[Constantin et al. 1994] P. Constantin, W. E, and E. S. Titi, "Onsager's conjecture on the energy conservation for solutions of Euler's equation”, Comm. Math. Phys. 165:1 (1994), 207-209. MR 96e:76025 Zbl 0818.35085

[Cozzi 2009] E. Cozzi, "Vanishing viscosity in the plane for nondecaying velocity and vorticity", SIAM J. Math. Anal. 41:2 (2009), 495-510. MR 2011a:76029 Zbl 05696712

[Cozzi 2010] E. Cozzi, "A finite time result for vanishing viscosity in the plane with nondecaying vorticity”, Commun. Math. Sci. 8:4 (2010), 851-862. MR 2012a:35226 Zbl 05843215

[Giga et al. 1999] Y. Giga, K. Inui, and S. Matsui, "On the Cauchy problem for the Navier-Stokes equations with nondecaying initial data", pp. 27-68 in Advances in fluid dynamics, edited by P. Maremonti, Quad. Mat. 4, Dept. Math., Seconda Univ. Napoli, Caserta, 1999. MR 2001g:35210 Zbl 0961.35110

[Giga et al. 2001] Y. Giga, S. Matsui, and O. Sawada, "Global existence of two-dimensional Navier-Stokes flow with nondecaying initial velocity", J. Math. Fluid Mech. 3:3 (2001), 302-315. MR 2002h:35236 Zbl 0992.35066

[Hmidi 2005] T. Hmidi, "Régularité höldérienne des poches de tourbillon visqueuses", J. Math. Pures Appl. (9) 84:11 (2005), 1455-1495. MR 2006i:35278 Zbl 1095.35024

[Kato 1972] T. Kato, "Nonstationary flows of viscous and ideal fluids in $\mathbf{R}^{3}$ ", J. Functional Analysis 9 (1972), 296-305. MR 58 \#1753 Zbl 0229.76018

[Kelliher 2004] J. P. Kelliher, "The inviscid limit for two-dimensional incompressible fluids with unbounded vorticity”, Math. Res. Lett. 11:4 (2004), 519-528. MR 2005g:76007 Zbl 1112.76007

[Masmoudi 2007] N. Masmoudi, "Remarks about the inviscid limit of the Navier-Stokes system", Comm. Math. Phys. 270:3 (2007), 777-788. MR 2008c:35234 Zbl 1118.35030

[Sawada and Taniuchi 2007] O. Sawada and Y. Taniuchi, "A remark on $L^{\infty}$ solutions to the 2-D Navier-Stokes equations", J. Math. Fluid Mech. 9:4 (2007), 533-542. MR 2009f:35255 Zbl 1132.35437

[Serfati 1995] P. Serfati, "Solutions $C^{\infty}$ en temps, $n$-log Lipschitz bornées en espace et équation d'Euler”, C. R. Acad. Sci. Paris Sér. I Math. 320:5 (1995), 555-558. MR 96c:35147 Zbl 0835.76012 
[Swann 1971] H. S. G. Swann, "The convergence with vanishing viscosity of nonstationary NavierStokes flow to ideal flow in $R_{3}$ ”, Trans. Amer. Math. Soc. 157 (1971), 373-397. MR 43 \#3662 Zbl 0218.76023

[Taniuchi et al. 2010] Y. Taniuchi, T. Tashiro, and T. Yoneda, "On the two-dimensional Euler equations with spatially almost periodic initial data", J. Math. Fluid Mech. 12:4 (2010), 594-612. MR 2012c:35337 Zbl 1270.35357

[Vishik 1999] M. Vishik, "Incompressible flows of an ideal fluid with vorticity in borderline spaces of Besov type”, Ann. Sci. École Norm. Sup. (4) 32:6 (1999), 769-812. MR 2000i:76008 Zbl 0938.35128

Received May 8, 2013. Revised October 31, 2013.

ELAINE COZZI

DEPARTMENT OF MATHEMATICS

OREGON STATE UNIVERSITY

368 KIDDER HALL

CORVALlis, OR 97331

UNITED STATES

cozzie@math.oregonstate.edu 


\title{
PACIFIC JOURNAL OF MATHEMATICS
}

\author{
msp.org/pjm
}

Founded in 1951 by E. F. Beckenbach (1906-1982) and F. Wolf (1904-1989)

\section{EDITORS}

Don Blasius (Managing Editor)

Department of Mathematics

University of California

Los Angeles, CA 90095-1555

blasius@math.ucla.edu

\author{
Paul Balmer \\ Department of Mathematics \\ University of California \\ Los Angeles, CA 90095-1555 \\ balmer@math.ucla.edu \\ Robert Finn \\ Department of Mathematics \\ Stanford University \\ Stanford, CA 94305-2125 \\ finn@math.stanford.edu \\ Sorin Popa \\ Department of Mathematics \\ University of California \\ Los Angeles, CA 90095-1555 \\ popa@math.ucla.edu
}

\author{
Vyjayanthi Chari \\ Department of Mathematics \\ University of California \\ Riverside, CA 92521-0135 \\ chari@math.ucr.edu \\ Kefeng Liu \\ Department of Mathematics \\ University of California \\ Los Angeles, CA 90095-1555 \\ liu@math.ucla.edu \\ Jie Qing \\ Department of Mathematics \\ University of California \\ Santa Cruz, CA 95064 \\ qing@ cats.ucsc.edu
}

\section{PRODUCTION}

Silvio Levy, Scientific Editor, production@msp.org

\section{SUPPORTING INSTITUTIONS}

ACADEMIA SINICA, TAIPEI

CALIFORNIA INST. OF TECHNOLOGY

INST. DE MATEMÁTICA PURA E APLICADA

KEIO UNIVERSITY

MATH. SCIENCES RESEARCH INSTITUTE

NEW MEXICO STATE UNIV.

OREGON STATE UNIV.

\author{
STANFORD UNIVERSITY \\ UNIV. OF BRITISH COLUMBIA \\ UNIV. OF CALIFORNIA, BERKELEY \\ UNIV. OF CALIFORNIA, DAVIS \\ UNIV. OF CALIFORNIA, LOS ANGELES \\ UNIV. OF CALIFORNIA, RIVERSIDE \\ UNIV. OF CALIFORNIA, SAN DIEGO \\ UNIV. OF CALIF., SANTA BARBARA
}

\author{
Daryl Cooper \\ Department of Mathematics \\ University of California \\ Santa Barbara, CA 93106-3080 \\ cooper@math.ucsb.edu \\ Jiang-Hua Lu \\ Department of Mathematics \\ The University of Hong Kong \\ Pokfulam Rd., Hong Kong \\ jhlu@maths.hku.hk \\ Paul Yang \\ Department of Mathematics \\ Princeton University \\ Princeton NJ 08544-1000 \\ yang@math.princeton.edu
}

These supporting institutions contribute to the cost of publication of this Journal, but they are not owners or publishers and have no responsibility for its contents or policies.

See inside back cover or msp.org/pjm for submission instructions.

The subscription price for 2014 is US $\$ 410 /$ year for the electronic version, and \$535/year for print and electronic.

Subscriptions, requests for back issues and changes of subscribers address should be sent to Pacific Journal of Mathematics, P.O. Box 4163, Berkeley, CA 94704-0163, U.S.A. The Pacific Journal of Mathematics is indexed by Mathematical Reviews, Zentralblatt MATH, PASCAL CNRS Index, Referativnyi Zhurnal, Current Mathematical Publications and Web of Knowledge (Science Citation Index).

The Pacific Journal of Mathematics (ISSN 0030-8730) at the University of California, c/o Department of Mathematics, 798 Evans Hall \#3840, Berkeley, CA 94720-3840, is published twelve times a year. Periodical rate postage paid at Berkeley, CA 94704, and additional mailing offices. POSTMASTER: send address changes to Pacific Journal of Mathematics, P.O. Box 4163, Berkeley, CA 94704-0163.

PJM peer review and production are managed by EditFLOW ${ }^{\circledR}$ from Mathematical Sciences Publishers.

\section{PUBLISHED BY}

\section{mathematical sciences publishers \\ nonprofit scientific publishing}

http://msp.org/

(C) 2014 Mathematical Sciences Publishers 


\section{PACIFIC JOURNAL OF MATHEMATICS}

Volume $270 \quad$ No. $2 \quad$ August 2014

Disjointification inequalities in symmetric quasi-Banach spaces and 257 their applications

SERgey Astashinin, Fedor A. SukocheV and DMitriy

ZANIN

Hamiltonian evolutions of twisted polygons in parabolic manifolds:

287

The Lagrangian Grassmannian

GLORIA MARÍ BEFFA

On Schwarz-Christoffel mappings

MARTIN CHUAQUi and CHRISTIAN POMMERENKE

Vanishing viscosity in the plane for nondecaying velocity and vorticity, II

ELAINE COZZI

Affine quantum Schur algebras and affine Hecke algebras

QIANG FU

On the classification of Killing submersions and their isometries

JosÉ M. MANZANO

Locally Lipschitz contractibility of Alexandrov spaces and its applications

Ayato Mitsuishi and TAKaO Yamaguchi

Sequences of open Riemannian manifolds with boundary

RAQUEL PERALES and CHRISTINA SORMANI

Invariant differential operators on a class of multiplicity-free spaces 